\title{
INVESTIGATING OF EFFECTIVE PARAMETERS ON STRESS BEHAVIOR OF PRESSURE DEEP TWIN TUNNELS UNDER PRESSURE
}

\author{
Mehdi Panji \\ Department of civil engineering, Zanjan Branch, Islamic Azad University, Zanjan, Iran \\ mehdipanji@iauz.ac.ir \\ Pouya Kavandi \\ Department of civil engineering, Zanjan Branch, Islamic Azad university*, Zanjan , Iran \\ Pouya.kavandi@yahoo.com
}

\begin{abstract}
The twin tunnels under the pressure have been analyzed in this study. Full-plane boundary element method (BEM) has been used for modeling. Focus of meshing on the boundary of twin tunnels distinguishes distinct features of the mentioned method with other numerical methods. In this regard, in addition to providing generalities of elastostatic formulation of the method and the required verification, the numerical study of the two tunnel has been done on stress and displacement components. The results showed that if the distance between two tunnel was more than 30R ( $\mathrm{R}$ radius of tunnel), the vertical stress on the wall of one of the twin tunnels was converged towards an alone tunnel.
\end{abstract}

Keywords: BEM, deep twin tunnels, stress behaviors, effective radius.

\section{INTRODUCTION}

Today tunnel construction is very important. The presence of conditions such as high traffic volume has increased the use of parallel tunnels. Digging two tunnels next to each other with less than a distance makes them influence on each other, and endangers the stability of tunnels. When the two tunnels are dug next to each other, they will interact with each other due to vicinity, and cause deformations. Thus, it is necessary to examine the effective of the second tunnel on the first tunnel. The increasing development of computer science and computer use for analysis due to the ease and high speed has created numerical methods in engineering. BEM and finite element method (FEM) can be mentioned one of the numerical methods. Several laboratory and numerical studies have been done on the effect of the two tunnels on each other. A study was done by Kim etal [1] based on laboratory studies in 1996. He concluded that the effect of tunnels on each other in two parallel tunnels leads to tunnel crown move downward. Liu etal [2] carried out three-dimensional analysis on parallel tunnels. Chen etal[3] has also studied the distance pillar on parallel tunnel's behavior in 2009. Following loganathan \& poulos [4] have offered analytical responses to determine the displacement of soft ground due to digging tunnel. A study conducted by Gioda and locatelli [5] in 1999 on the effective of digging tunnel in sandy land with the help of field measurements. In 2003, displacement ground for an environment with two tunnels was investigated using centrifuge test [6]. For tunnels modeling in various geometric states, material properties were performed using FEM by Koutasbeleulis \& giriffith [7]. Lee and Rowe [8] investigated around the tunnel in clay soils using FEM deformation.

Beginning of BEM formation is nearly four decades. However, significant progresses have happened in the development of boundary element method. Discrete process of the boundary was used for the first time by Fredholm [9] in 1903 for potential flow equations. Since 1980 BEM was used to solve the soil problems, so that some researchers investigated discrete method of boundary in unbounded space [10 and 11]. In 2011, Duenser etal[12] simulated the underground structures using boundary element method, and analyzed their behavior, Also in 2006 so far Panji etal[13,14,15 and 16] have studied basic solutions of the full \& half page using numerical method, and have utilized it to analyze 
foundation and tunnel. The aim of the research is to examine the most minimum distance between two tunnels that do not effective on each other and is a state similar to absence of the second hole.

\section{FULL-PLANE BEM}

One of the most important features of BEMmodeling of semi-infinite environments is without considering approximation. In numerical methods such as FEMto model the infinite and semi-infinite environments, it is required to analyze the scope with regarding an approximation from the environment. This will reduce the accuracy of these methods. Also in the case of extensive environment, the volume of calculations is increased, and finally the results are achieved slowly.

In boundary element method only with discrete boundary loading in infinite and semi-infinite environments, the whole environment is participated in the analysis, that in addition to higher speed it will have also higher accuracy. Tunnels in infinite space, loading on the surface of soil, wide strip surface or deep foundations with considering soil-structure interaction effect can be mentioned of a variety of infinite and semi-infinite environments.

\section{FORMULATION OF BOUNDARY ELEMENTS IN FULL PLANE}

By applying the weighted residuals' integral on equation of Navier elastostatic balance regardless of volume forces, we will have [17].

$$
\int_{\Omega} \Omega_{k j . j} \mathrm{u}_{\mathrm{k}}^{*} \mathrm{~d} \Omega=0
$$

So that in the above equation, $\mathrm{u}_{\mathrm{k}}^{*}$ is weight function in weighted residuals or full-space Kelvin fundamental solution, $\Omega$ represents the problem, and $\sigma_{k j . j}$ is the equilibrium equation according to stress components. Solving the fundamental displacement in two-dimensional elasticity utilizing Navier equation and Galerkin method is obtained as follows:

$$
u_{l k}^{*}=\frac{1}{8 \pi \mu(1-v)}\left[(3-4 v) \ln \frac{1}{r} \delta_{l k}+r_{, l} r_{, k}\right]
$$

$\mathrm{u}_{\mathrm{k}}^{*}$ is full space displacement fundamental solution along 1 under a single force along r.k $=1.2 .1=1.2$ ., $\mathrm{r}$ is distance of source point to resiver point (boundary or internal), $\mathrm{r} 1$ is derivative of $\mathrm{r}$ vector compared to along $1 \delta_{\mathrm{IK}}, l$ is Kronecker delta, and $v, \mu$ represent the shear modulus and Poisson's coefficient, respectively. Twice part-by-part integration of equation (1), the Green relation is obtained as fallows:

$$
\int_{\Omega} \sigma_{k j . j}^{*} \mathrm{u}_{\mathrm{k}} \mathrm{d} \Omega=-\int_{\Gamma} p_{\mathrm{k}} \mathrm{u}_{k}^{*} \mathrm{~d} \Gamma+\int_{\Gamma} u_{\mathrm{k}} \mathrm{p}_{k}^{*} \mathrm{~d} \Gamma
$$

So that $\mathrm{p}_{k}$ and $u_{\mathrm{k}}$ represent the values of traction and boundary displacement of boundry. $\mathrm{p}_{k}^{*}$ Is fundamental solution of full space traction and $\Gamma$ specifies boundary. Using Dirac delta function (one of the Boundary solution methods) boundary integral equation (BIE) is offered after the removal of sentences over the domain as follows:

$$
c_{I k}^{i} u_{l}^{i}+\int_{\Gamma} p_{l k}^{*} u_{k} d \Gamma=\int_{\Gamma} u_{l k}^{*} d \Gamma
$$

In the above equation $c_{i k}^{\mathrm{i}}=1-\left(\theta_{1} / 2 \pi\right)$ and $\theta_{1}$ are placement angle of boundary node of $\mathrm{i}$ (angle of boundary refraction). Fundamental solution of traction components of $p_{l k}^{*}$ is achieved as follows:

$$
p_{l k}^{*}=-\frac{1}{4 \pi(1-v) r}\left[\begin{array}{c}
\frac{\partial r}{\partial n}\left[(1-2 v) \delta_{I K}+2 r_{j} r_{k}\right] \\
+(1-2 v)\left(n_{j} r_{k}-n_{k} r_{j}\right)
\end{array}\right]
$$


In this relation, $n_{j}$ is jth-component of normal vector perpendicular to the boundary of $\Gamma$ and $\partial r / \partial n$ is derivative of position vector of $\mathrm{r}$ compared to the normal vector. In the used of fundamental solutions of $u_{l k}^{*}$ and $p_{l k}^{*}$, as well as BIM of 4 , boundary unknowns can be obtained for each boundary node of $i$. It should be noted that stress fundamental solutions to determine the stress at each internal point defined in the problem can be achieved using equilibrium equation and fundamental solutions of full space displacement as follows:

$$
\begin{aligned}
& \sigma_{i j}=\int_{\Gamma} D_{k i j}^{*} p_{k} d \Gamma-\int_{\Gamma} S_{k i j}^{*} u_{k} d \Gamma \\
& D_{k i j}^{*}=\frac{1}{r}\left\{\begin{array}{c}
(1-2 v)\left\{\begin{array}{c}
\left.\delta_{k i} r_{, i}+\delta_{k i} r_{, i}-\delta_{i j} r_{, k}\right\} \\
+2 r_{, i} r_{, j} r_{, k}
\end{array}\right\} \frac{1}{4 \pi(1-v) r} \\
S_{k i j}^{*}=\frac{2 \mu}{r^{2}}\left\{2 \frac{\partial r}{\partial n}\left[\begin{array}{c}
(1-2 v) \delta_{i j} r_{k} \\
+v\left(\delta_{i k} r_{j}+\delta_{j k} r_{j}\right)-4 r_{j} r_{j} r_{k}
\end{array}\right]+2 v\left(n_{i} r_{, j} r_{, k}+n_{i} r_{, j} r_{, k}\right)\right)+ \\
\left.\quad(1-2 v)\left(2 n_{k} r_{, j} r_{, j}+n_{j} \delta_{i k}+n_{i} \delta_{j k}\right)-(1-4 v) n_{k} \delta_{i j}\right\} \cdot \frac{1}{4 \pi(1-v)}
\end{array}\right.
\end{aligned}
$$

In the above equations $D_{k i j}^{*}$ and $S_{k i j}^{*}$ are fundamental solutions of internal stress and $\delta_{y}$ is the stress tensor.

\section{NUMERICAL MODELING}

First, a software was prepared based on full-plane BEM which was presented in the above. This software is able to analyze and examine the subsurface tunnels in a static state. In the Figure 1, a numerical study including geometric parameters and material properties was shown. Also, the discretized boundaries can be seen in this figure. 


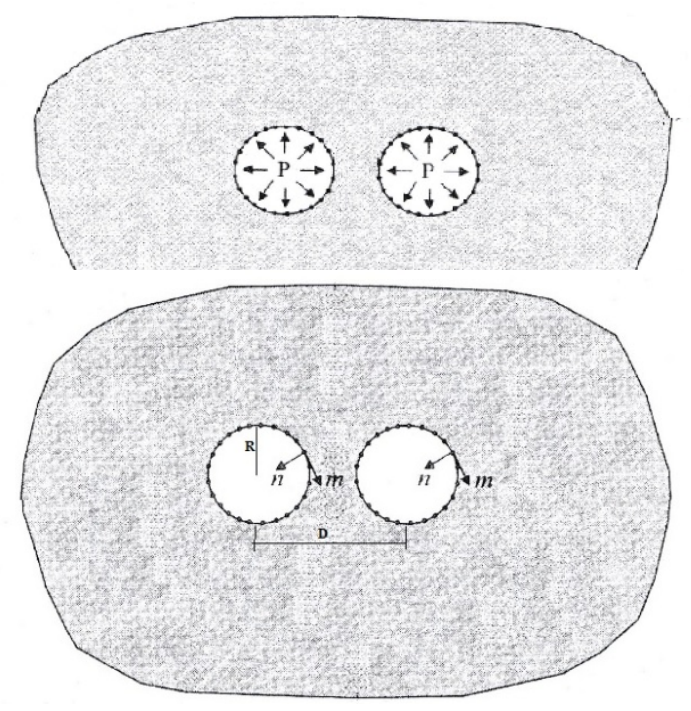

Figure 1: the modeling deep twin tunnel under pressure for present study

\section{ASSUMPTIONS}

The assumptions of present study are presented as follows:

1. Radius of the tunnel in this study has been assumed $3 \mathrm{~m}$.

2. Poisson's ratio has been considered 0.1.

3. Quadratic element has been used for discrediting the tunnel boundary.

4. pressure value has been assumed $100 \mathrm{MPa}$.

\section{VERIFICATION}

In this state, first a tunnel has been investigated under the pressure of $100 \mathrm{MPa}$ in a static state Figure2. Then, by creating a second tunnel parallel to the first tunnel, stress disorganization and stress change can be seen around the first tunnel. In this study, it has been tried to make zero the effective of second tunnel on the first one by increasing distance. According to the results obtained from this program that have been shown in Figure 2, it can be seen that the effective of chart with distance more than $30 \mathrm{R}$ has become zero. 


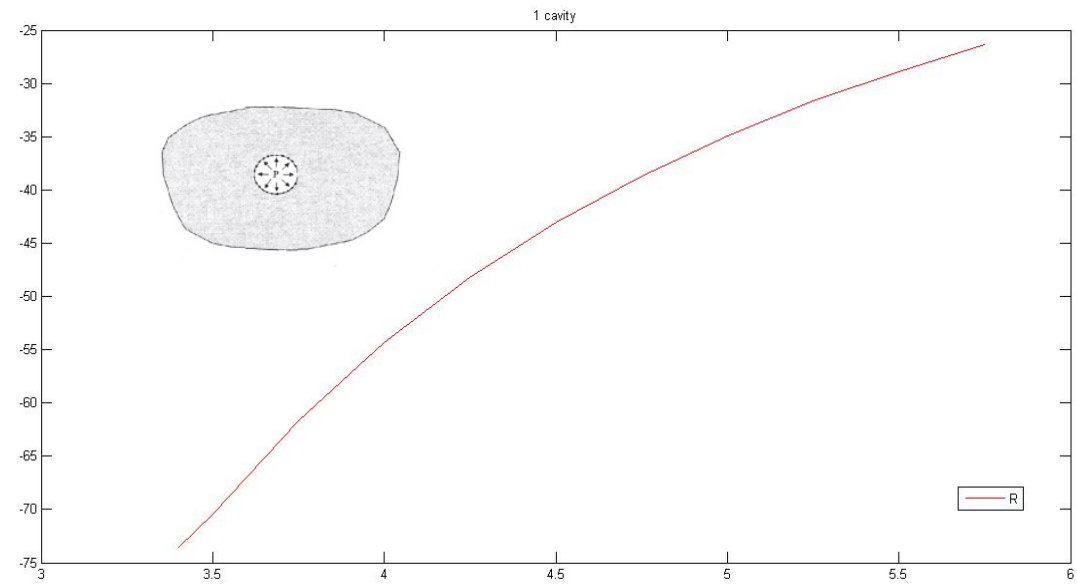

Figure 3: First tunnel without the second tunnel

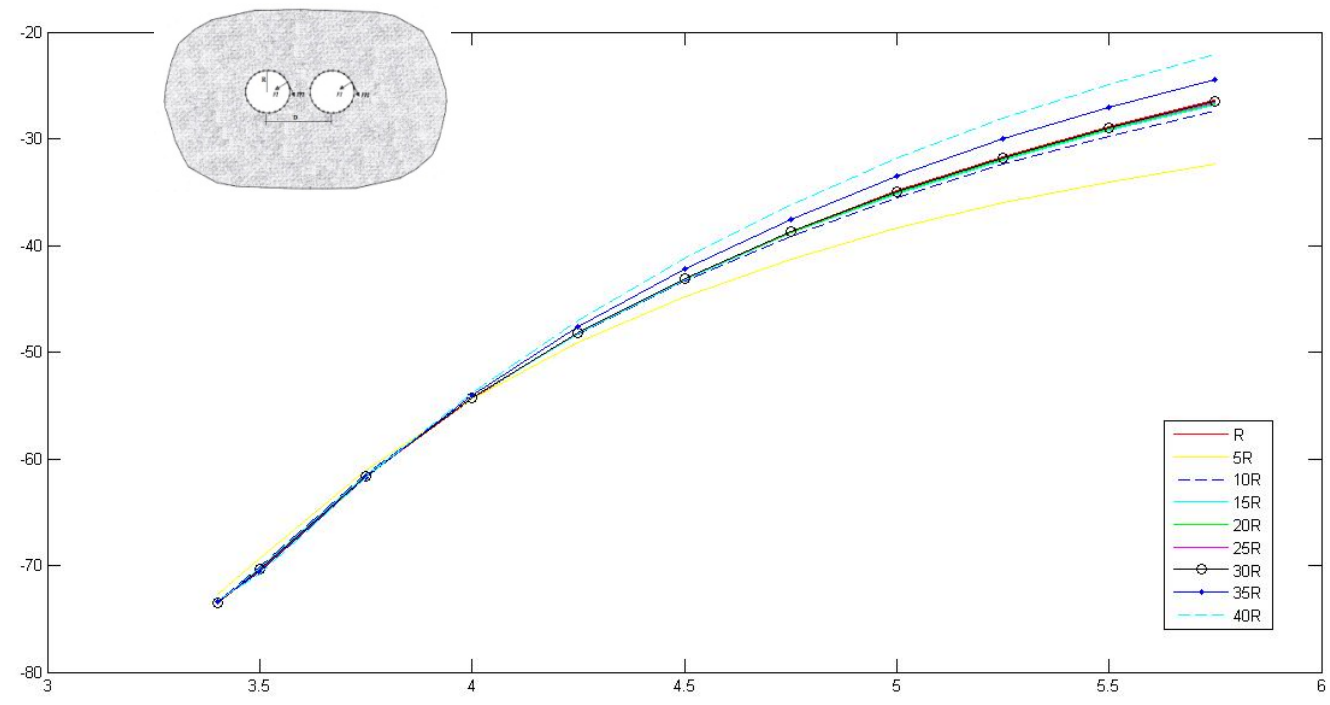

Figure 4: Diagram of twin tunnels

\section{VERIFICATION EXAMINATION}

Today authenticity of a subject in engineering is done in two ways. The first method is examination of the current method with previous researchers' studies that in addition it should not violated them, should have more accuracy than them. The later method mentions an engineering reason to prove the subject. According to Figure 4, it can be seen that increasing the distance between the two tunnels from other reduces the effective of the second tunnel on first tunnel. This is a logical reason and confirms the authenticity of the software.

\section{CONCLUSION}

According to conducted analysis using the prepared algorithm, the importance of the presence of two parallel tunnels on stress responses and their displacement was analyzed. The results showed that the effective radius on each other is removed and the responses of each of the tunnels converge towards 
the single hole tunnel by increasing distance of one of the tunnels to the other tunnel as much as 30 times the tunnel radius.

\section{REFERENCES}

Kim SH, Burd HJ, Milligan GWE (1998). "Model testing of closely spaced tunnels in clay". Geotechnique 48 (3): 375-388.

Liu, H.Y; Small, J.C; Carter, J.P. (2007) "Full 3D Modeling for Effects of Tunneling on Existing Support System in the Sydney Region". J. Elsevier, Tunneling and Underground Space Technology. 23, PP 399- 420.

Chen, Shong-loong; LI, Guo-wei; GUI, Meen-wah. "Effects of overburden, rock strength and pillar width on the safety of a three-parallel-hole tunnel" .j. Springer, Journal of Zhejiang University SCIENCE, 2009, PP 1581-1588.

Loganathan, N. and Poulos, H. G. (1998) "Analytical prediction for tunneling induced ground movements in clays", J. Geotech. Geoenviron. Eng., Pp.846-856.

Gioda, G. and Locatelli, L. (1999) "Back analysis of the measurements performed during the excavation of a shallow tunnel in sand", Int. J Numer. Anal. Meth. Geomech, Vol. 23, No. 13, pp.1407-1425.

Wu, D. R. and Lee, C. J. (2003) "Ground movements and collapse mechanism induced by

Koutasbeloulis, N. C., Griffiths, D. V. (1989) "Numerical modeling of the trap door problem", Geotechnique 39, pp.77-89.

Lee, K. M. and Rowe, R. K. (1991) "An analysis of three-dimensional ground movements: the Thunder Bay Tunnel", Can. Geotech. J., Vol. 28, pp.25-41.

Fredholm. I. (1903) "Sur une classe d'equations fonctionelles", Acta Math. 27, pp.365-390.

Banerjee, P.K. and Butterfield, R. (1981); "Boundary Element Methods in Engineering Science", McGraw- Hill Press, London.

Crouch, S.L. and Starfield, A.M. (1983), "Boundary Elements Method in Solid Mechanics", UNWIN HYMAN Inc., London.

Duenser, C., Thoeni, K., Riederer, K., Lindner, B and Beer, G. (2011) "New developments of the boundry element method for underground constructions", International Journal of Geomechanics, No.10, pp.1061-1097.

Panji, M. (2007) "The stress analysis of infinite and semi-infinite continuum media using boundary element methods", Msc Thesis, Islamic Azad University, Central Tehran Branch, Tehran, Iran.

Asgari Marnani, J. \& Panji, M. (2006) "The behavior of the clay soils on the strip foundation to eccentric load," the scientific-promotional magazine 44, 29 and 30, Page 34

Asgari Marnani, J. \& Panji, M. (2007) "Boundary element methods, theory and applications", second edition publishing Newshangar, Iran.

Panji, M., Asgari Marnani, J. and Tavousi Tafreshi, SH. (2011) "Evaluation of effective parameters on the underground tunnel stability using BEM", Journal of Structural Engineering and Geotechnics, Vol. 1, No. 2, pp.29-37.

Panji, M. Asgari Marnani, J. Ali Elahi, h. Koohsar h. Adampira M, (2013) " evaluation of effective parameters on stress behavior of 2D shallow tunnels using boundary elemnt method " Journal of pajoheshname hamlonaghl, Vol. 10, No.1, pp.17-28. 